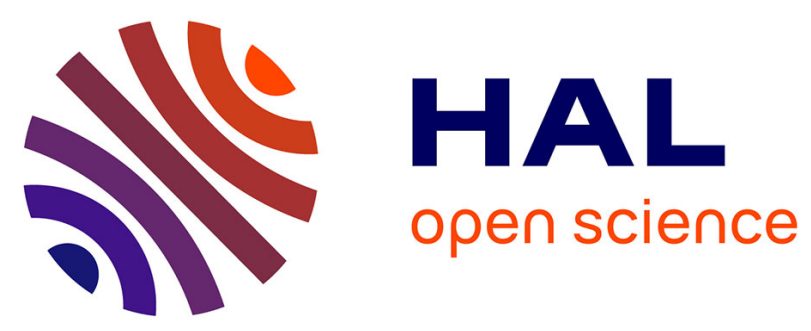

\title{
Calorimetric analysis of polymer behaviour using a pixel calibration of an IRFPA camera
}

Vincent Honorat, Stéphane Moreau, Jean Michel Muracciole, Bertrand Wattrisse, Andre Chrysochoos

\section{To cite this version:}

Vincent Honorat, Stéphane Moreau, Jean Michel Muracciole, Bertrand Wattrisse, Andre Chrysochoos. Calorimetric analysis of polymer behaviour using a pixel calibration of an IRFPA camera. Quantitative InfraRed Thermography Journal, 2005, 2 (2), pp.153-172. 10.3166/qirt.2.153-171 . hal-00572562

HAL Id: hal-00572562

https://hal.science/hal-00572562

Submitted on 22 Nov 2021

HAL is a multi-disciplinary open access archive for the deposit and dissemination of scientific research documents, whether they are published or not. The documents may come from teaching and research institutions in France or abroad, or from public or private research centers.
L'archive ouverte pluridisciplinaire HAL, est destinée au dépôt et à la diffusion de documents scientifiques de niveau recherche, publiés ou non, émanant des établissements d'enseignement et de recherche français ou étrangers, des laboratoires publics ou privés. 


\section{Calorimetric analysis of polymer behaviour using a pixel calibration of an IRFPA camera}

\section{Honorat - S. Moreau - J.-M. Muracciole - B. Wattrisse - A. Chrysochoos}

Laboratoire de Mécanique et Génie Civil, UMR 5508 CNRS-UMII

Université Montpellier II, Place E. Bataillon

34095 Montpellier Cedex 05

(chryso@lmgc.univ-montp2.fr)

ABSTRACT: A pixel calibration of an IRFPA camera was developed to detect very small temperature variations induced by quasi-static loading of materials. The thermographic data were then used to estimate heat sources accompanying the deformation of PMMA and PC polymers during cyclic tests. The calorimetric balance analysis led us to define several possible ways of introducing the thermoelastic coupling effects in viscothermoelastic models.

KEY WORDS: thermography / IRFPA camera calibration / polymers / viscothermoelasticity / calorimetric balance / 


\section{Introduction}

Temperature variations of solid materials induced by quasi-static mechanical loadings may remain low. The amplitude of these variations also decreases when the heat capacity and/or the conductibility increases. From a theoretical standpoint, these experimental facts implicitly warranted the development of isothermal behavioural models. Nevertheless, temperature variations, irrespective of how small they are, may represent amounts of heat that should be taken into account in the energy balance and consequently in the thermomechanical model construction.

We thus developed a specific calibration protocol for an infrared focal plane array (IRFPA) camera to detect homogeneity defects of about $20 \mathrm{mK}$. The method substitutes a calibration of each pixel for the "mean" calibration of the thermo signal as proposed by the camera designer (Cedip).

We recently used this calibration method for studying the thermomechanical behaviour of two vitreous polymers, i.e. the polymethyl metacrylate (PMMA) and the polycarbonate (PC). In order to draw up complete energy balances associated with the deformation process, it was essential to determine the capabilities of our calibration protocol to be able to accurately estimate the intensity and distribution of heat sources developed during uniaxial cyclic tests.

Note that, generally speaking, polymers are considered as viscoelastic materials within the framework of small, quasi-static perturbations (Alfrey 48), (Biot 65), (Ferry 80). In what follows, we will show that the thermal and calorimetric behaviour of the above-mentioned polymers led us to consider linear viscothermoelastic models instead of purely viscoelastic models (Moreau 04). Our analysis of the mechanical and calorimetric effects indeed showed that for small deformations $\left(<2.10^{-2}\right)$ and low strain rates $\left(<5.10^{-4} \mathrm{~s}^{-1}\right)$, i.e. low stress levels $(<50 \mathrm{MPa})$, the intensity of the intrinsic dissipation $d_{1}$ remained negligible compared to the thermoelastic source amplitude $\Delta \mathrm{s}_{\text {the }}\left(d_{1} \approx \Delta \mathrm{s}_{\text {the }} / 30\right)$. Moreover, our experiments showed that the thermoelastic effects could vary between polymers under the same testing conditions.

This paper first presents the rationale that prompted us to develop a pixel calibration protocol.

Secondly, it indicates how the viscothermoelastic extension of two basic viscoelastic models may give different thermoelastic responses under the same loading conditions.

Thirdly, based on a thermographic analysis of calorimetric effects, thermoelastic sources induced by the deformation of PMMA and PC polymers are finally shown to respectively be in quite good agreement with predictions generated by extended Poynting-Thomson (PT) and Zener (Z) type models. 


\section{Calibration protocol}

The experimental set up involved a testing machine (frame $100 \mathrm{kN}$, load cell +/$25 \mathrm{kN}$ ) and an IRFPA camera. The camera used in this work was a Cedip Jade MWIR 3-5.2 $\mu \mathrm{m}$. The focal length of the optical lens is $25 \mathrm{~mm}$. This camera is equipped with an InSb $320 \times 240$ element detector, cooled at $80 \mathrm{~K}$ with a Stirling device. The lens axis of the camera was kept fixed and held perpendicular to the surface of the specimen during the mechanical tests.

The basic sketch of the thermography workstation is shown in Figure 1. The IR influx passing through the lens is converted into an electrical tension signal by the detector. An analog-to-digital converter (ADC) then provides a digital signal coded in digital levels (DL).

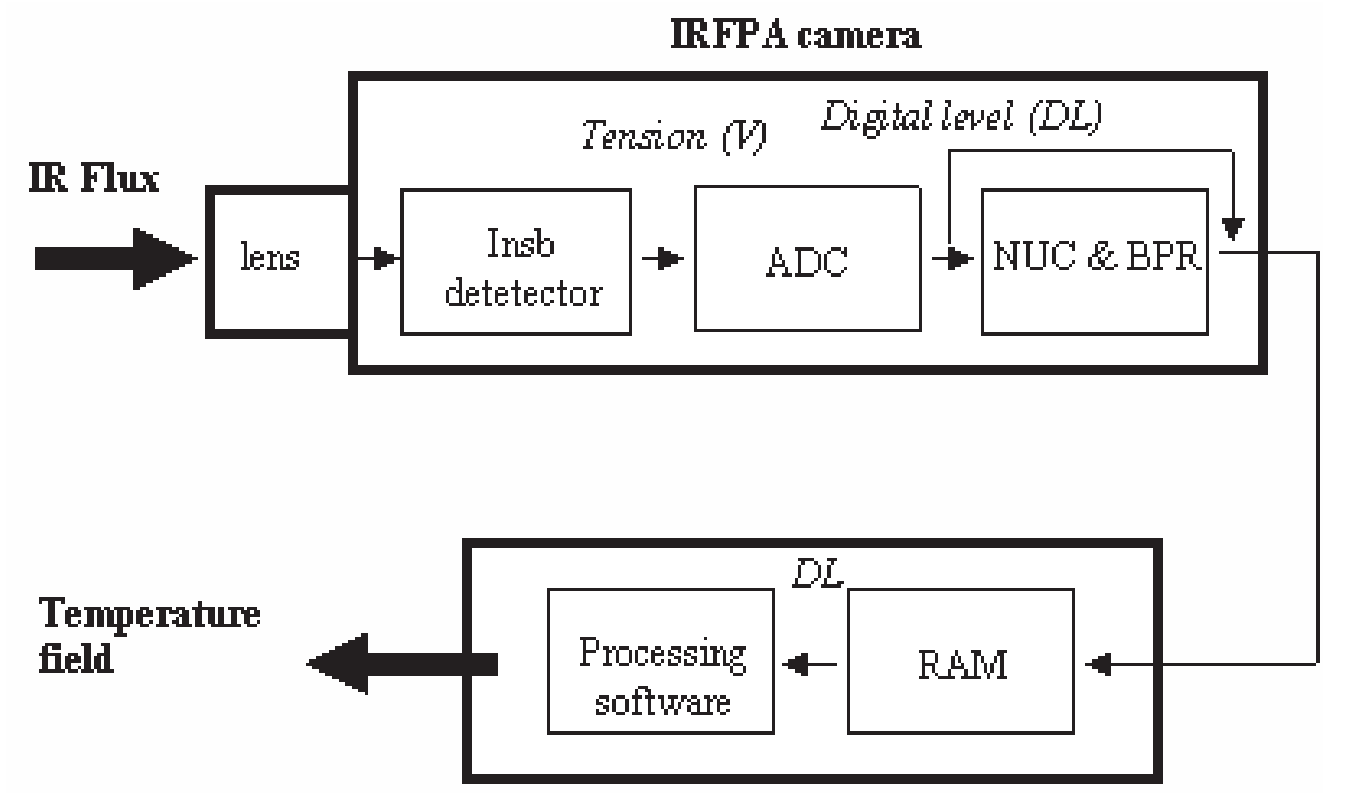

Processing software

Figure 1. Basic sketch of the thermography workstation

The next stage takes the non-uniform response of the detector elements when placed in front of a uniform radiative source into account. This is the non-uniformity correction (NUC) operation. At this stage, the so-called "bad pixels" are also tracked and replaced. This is the bad pixel replacement (BPR) operation. These basic operations are part of the standard calibration protocol. They will be summarised and described hereafter. The digital video is finally stored in the RAM of the computer. A software package developed by the camera designer then enables calibration, processing and visualization of thermal data.

A stable and spatially uniform IR radiation source is used to perform NUC, BPR and camera signal calibration. We therefore used a planar black body SR $80-4 \mathrm{~A}$ (by CI Systems) with the following main specifications: accuracy $\left(+/-0.008^{\circ} \mathrm{C}\right.$ for $5^{\circ} \mathrm{C}$ temperature amplitude), thermal resolution $\left(\delta T_{R}=0.01^{\circ} \mathrm{C}\right)$, and spatial nonuniformity of the target (less than $+/-0.02^{\circ} \mathrm{C}$ ). 


\subsection{Standard calibration protocol}

\subsubsection{Non-uniformity correction}

As mentioned above, each sensor of the matrix has its own response. For one element of the detector and for "moderate" IR radiation fluxes (i.e. not close to "low" or "high" saturation of the detector element, see Figure 2), the correspondence between flux and tension is linear. This is why the camera designer proposes a linear fit of the detector mean signal. The standard calibration uses two uniform "cold" and "hot" sources provided by the black body. The thermo signal is the mean digital level delivered by the detector for a given source temperature. For two temperatures $T_{1}$ and $T_{2}$ of the black body, the corresponding values of the detector response are respectively:

$$
\begin{aligned}
& S_{1} \square \frac{1}{I} \sum_{i \square 1}^{I} s_{i 1}, \\
& S_{2} \square \frac{1}{I} \sum_{i \square 1}^{I} s_{i 2},
\end{aligned}
$$

where $I$ is the number of elements of the detector and $s_{\mathrm{ij}}$ the digital signal delivered by the $i^{\text {th }}$ element at temperature $T_{\mathrm{j}}$.

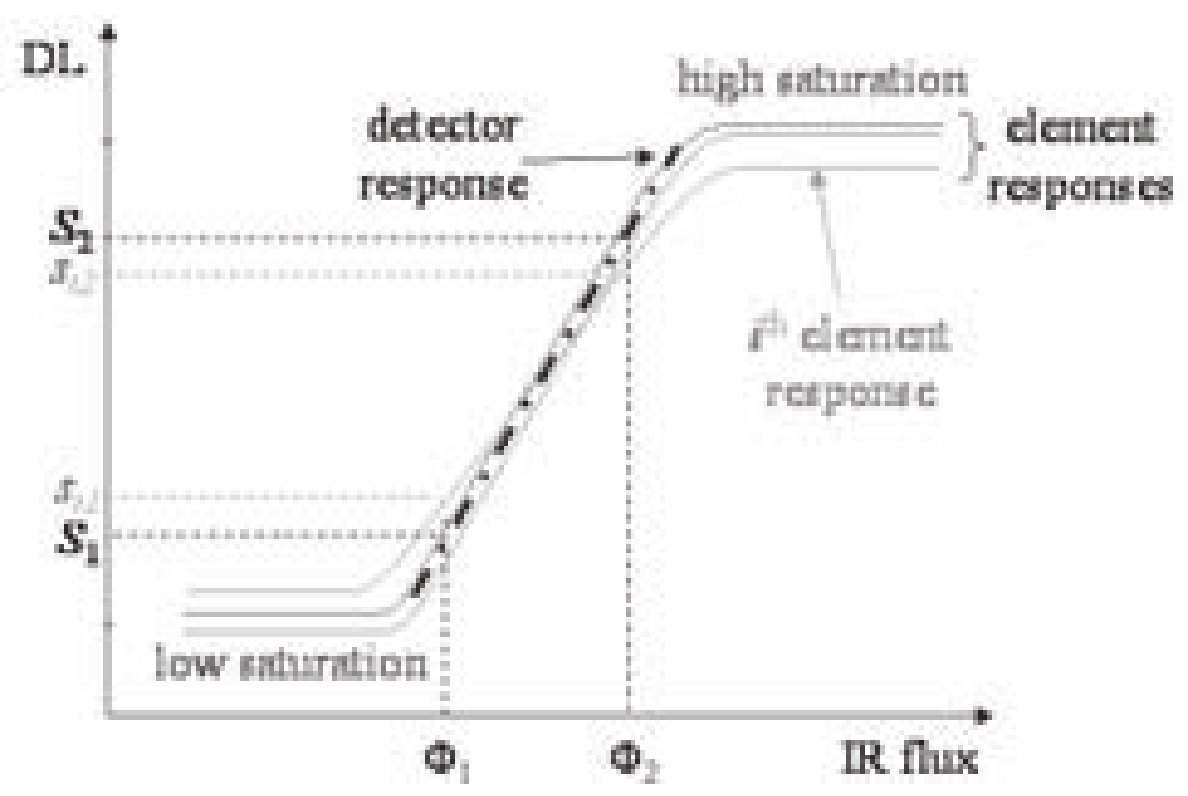

Figure 2. Non-uniformity correction

To get a uniform response of the detector elements for all uniform thermal scenes, gain $\alpha_{i}$ and offset $\beta_{i}$ are then computed for each element:

$$
\alpha_{i} \square \frac{S_{2}-S_{1}}{s_{i 2}-s_{i 1}}, \quad i \square 1,2, \ldots, I,
$$




$$
\beta_{i}=S_{1}-\alpha_{i} s_{i 1}, \quad i=1,2, \ldots, I .
$$

Indeed, if we define $\tilde{s}_{i j} \square \alpha_{i} s_{i j} \square \beta_{i}$ (without summation over $i$ ) for a temperature $T_{j}$ limited to $T_{j} \in\left[T_{1}, T_{2}\right]$, the reader can easily verify that:

$$
\left\{\begin{array}{l}
\tilde{s}_{i j} \square S_{2} x_{i j} \square S_{1}\left(1-x_{i j}\right) \\
x_{i j} \square \frac{s_{i j}-s_{i 1}}{s_{i 2}-s_{i 1}}
\end{array} .\right.
$$

Equation 5 clearly shows that the detector response remains uniform for a uniform source as long the response of each element remains linear (see Figure 2). Note that although the use of polynomials of higher degree would likely not improve the measurement accuracy, this operation would make it possible to take the nonlinear response of some detector elements whose state is near saturation into account. According to the camera designer, the optimal calibration is obtained for a couple of temperatures $\left(T_{1}, T_{2}\right)$ corresponding to $30 \%$ and $70 \%$ of the digital detector range (Figure 2). In this case, the calibration error will be less than the noise equivalent temperature difference $(\mathrm{NETD}=20 \mathrm{mK})$.

\subsubsection{Bad pixel replacement}

When the response $s_{i}$ of the $i^{\text {th }}$ element is not "close" enough to the mean response $S$, this element is supposed to be defective and is identified as a bad pixel. Different criteria have been proposed by Cedip to define a bad pixel:

- Responsivity method: If the response of all detector elements were the same, all $\alpha_{i}$ would be equal to 1 . Then the system will consider a pixel as bad if the gain coefficient $\alpha_{i}$ is lower or higher than a predefined percentage set by the user. For instance, for a given threshold $a>0$, bad pixels are those for which $\left|\alpha_{i}-1\right| \square a \square$

- Offset method: If the detector elements had the same response, all $\beta_{i}$ would be equal to $0 \mathrm{DL}$. The system will consider a pixel as bad if the offset coefficient $\beta_{i}$ is lower or higher than a predefined threshold. For instance, if the threshold is $b>0$ and if the range of digitization is $R_{d}=2^{14} \mathrm{DL}$, the system will classify a pixel as bad if $\left|\beta_{i}\right| \square b R_{d}$.

- Noise method: the system will consider a pixel as bad if the rms noise is lower or higher than a predefined threshold. This threshold is derived from the mean and standard deviation of a noise image. For instance, if the threshold multiplier is $\xi \square \square$ and the mean and standard deviation are respectively $S$ and std, the system will classify a pixel as bad if the rms noise $N_{\mathrm{rms}}>S+\xi$ std. The rms noise of the $i^{\text {th }}$ element is defined for a shot of $M$ images captured at times $t_{m}$ by: 


$$
N_{r m s} q_{i} \square \sqrt{\frac{1}{M} \sum_{m \square 1}^{M}\left(\tilde{s}_{i}\left(t_{m}\right)-\bar{s}_{i}\right)^{2}}
$$

where $\bar{s}_{i}=\frac{1}{M} \sum_{m=1}^{M} \tilde{s}_{i}\left(t_{m}\right)$.

For each criterion, the same BPR procedure is proposed by Cedip (see Figure 3). For instance, if pixel 0 is bad, it is replaced by pixel 1, or by pixel 2 if pixel 1 is also bad, ... and so on.

\begin{tabular}{|c|c|c|c|c|}
\hline 21 & 13 & 9 & 14 & 22 \\
\hline 20 & 5 & 1 & 6 & 15 \\
\hline 12 & 4 & 0 & 2 & 10 \\
\hline 19 & 8 & 3 & 7 & 16 \\
\hline 24 & 18 & 11 & 17 & 23 \\
\hline
\end{tabular}

Figure 3. Bad pixel replacement algorithm

\subsubsection{Digital signal calibration}

Signal calibration can be carried out once the NUC and BPR operations are performed. This calibration should theoretically be based on Planck's law. Indeed, for a quasi-monochromatic detector placed in front of a uniform black body at temperature $T$, Planck's law is used to derive the following expression of the camera signal (Papini et al., 94):

$$
S \square \gamma \frac{C_{1} \lambda^{-5}}{\exp \left(\frac{C_{2}}{\lambda T}\right)-1} \square U_{0}
$$

where $\lambda$ is the mean wavelength of the spectral range of the detector, while $C_{1}, C_{2}, \gamma$ and $U_{0}$ are constants. Designers and offices of standardization and metrology generally prefer simplified versions of equation 7 . They also often choose a simple fitting of the curve $S(T)$ by a polynomial of degree 2 or 3, especially when small thermal ranges $\left[T_{1}, T_{2}\right]$ are considered.

$$
S(T) \square \sum_{p \sqsubset 0}^{P} a_{p} T^{p}, P \square 2 \text { or } 3, T \in T_{1}, T_{2} \sqsubset
$$

The polynomial coefficients $\left(a_{p}, p=0,1, . ., P\right)$ are classically derived from a leastsquares fit of $\left(S_{j}, T_{j}\right)$ couples $(j=1, . ., J)$. The number $J$ of thermal equilibrium states 
must be much greater than $P$. Besides, the temperatures $T_{j}$ have to be regularly distributed within $\left[T_{1}, T_{2}\right]$ to get a reliable fit.

\subsubsection{Discussion}

The major interest of the procedure described above is, in our opinion, its simplicity of use. The NUC table (gain +offset tables), the bad pixel list and the coefficients of the calibration law can be automatically managed by software. Different calibration files can be saved corresponding to different parameter sets of the camera (frame size, integration time, optional filter, etc.).

Nevertheless, this calibration is based on a homogenization of the detector response, whereas pixel responses are generally heterogeneous. All is done to get a uniform signal of the detector elements when the detector is placed in front of a uniform source. This enables the user to identify (and apply) a single calibration law inasmuch as $\tilde{s}_{i}(T) \square S(T), \forall i=1,2, \ldots, I$. We have already underlined that the NUC operation, as proposed in equation 5 , supposes a linear response of each detector element. First, this condition may limit the thermal range used for the calibration or may induce uncontrolled errors if some elements start to behave nonlinearly. Secondly, even if all element responses remain linear, the standard calibration protocol replaces some bad pixels because their responses are far from the mean detector response. In the framework of our thermomechanical applications, this replacement operation may distort the thermal gradient computation by spatially correlating measurement errors. This distortion consequently affects the heat source estimate.

These are the main reasons why we have proposed a new protocol based on an individual calibration of detector elements.

\subsection{Pixel calibration protocol}

It is possible to shortcut NUC and BPR operations with the IR thermography workstation used in this study (see Figure 2). This feature was used to develop an individual pixel calibration based on polynomial fitting of the digital level $s_{i}$ delivered by the $i^{\text {th }}$ element of the detector (equation 9) when the camera is placed in front of the black body source at different temperatures $T$,

$$
s_{i}(T) \square \sum_{p \sqsubset 0}^{P} a_{i p} T^{p} \quad \text { for } T \in T_{1}, T_{2}[.
$$

The $a_{i p}$ coefficients are derived from a least-squares fit. The user may first define the degree $P$ of the polynomial fitting function. Generally polynomials of degree $\mathrm{P} \geq 5$ are chosen. The user defines a number $J$ of equilibrium thermal states $T_{j}$ distributed between $T_{1}$ and $T_{2}$. 
The calibration protocol is partly managed by computer. Once the data are fitted, defective pixels are detected by using the following criterion. The system will consider pixel $i$ as a bad one if the difference between the temperature $T_{i j}$ (predicted by the polynomial fitting) and the ordered temperature of the black body source $T_{j}^{B B}$ is greater than a predefined threshold $\delta T$. This threshold is typically about $\delta T=40 \mathrm{mK}$ and is greater than the black body specifications. This criterion can be written as:

$$
\sup _{j=1,2, . ., J}\left|T_{i j}-T_{j}^{B B}\right| \geq \delta T
$$

To illustrate this criterion, Figure 4 shows a histogram characterizing the response of the pixel matrix for a given black body temperature $T^{B B}$. It indicates the number of pixels giving the same difference $\left(T_{i j}-T^{B B}\right)$. This error depends on:

- the temperature range $\left[T_{1}, T_{2}\right]$ used in the fitting operation

- the degree $P$ of the polynomial fitting function

- $\quad$ the camera parameter set: integration time, frame size, focal length, etc.

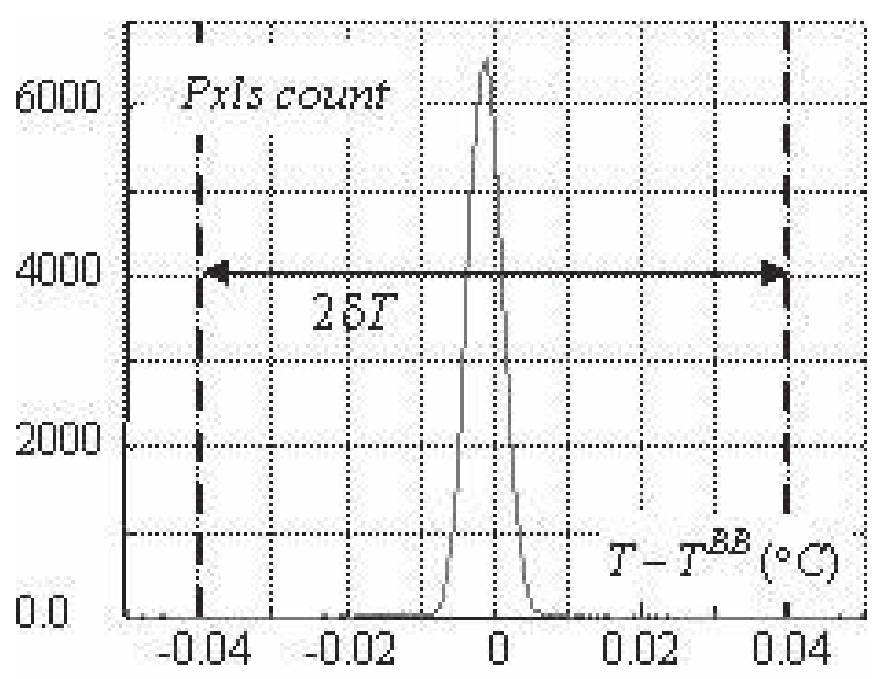

Figure 4. Histogram of pixel temperature differences at fixed $T^{B B}\left(17^{\circ} \mathrm{C}\right)$. Integration time $I T=2 \mathrm{~ms}$, full frame, focal length $=25 \mathrm{~mm}$.

The distribution of pixel responses looks like a non-centred Gaussian law. The number of pixels whose response is outside of the threshold band is low. For example, in the case of Figure 4 , we calculate $0.2 \%$ of bad pixels.

Figure 5 indicates where these pixels (circle) are located in the CCD matrix. Note that they are rather well distributed over the detector surface. Nevertheless we observe several bad pixel packs. As already mentioned, the BPR operation may substantially distort the thermal gradient calculations in these regions. For this 
reason, when a pixel is considered as bad, it is no longer taken into account in the continuation of the image processing (filtering and heat source computation).

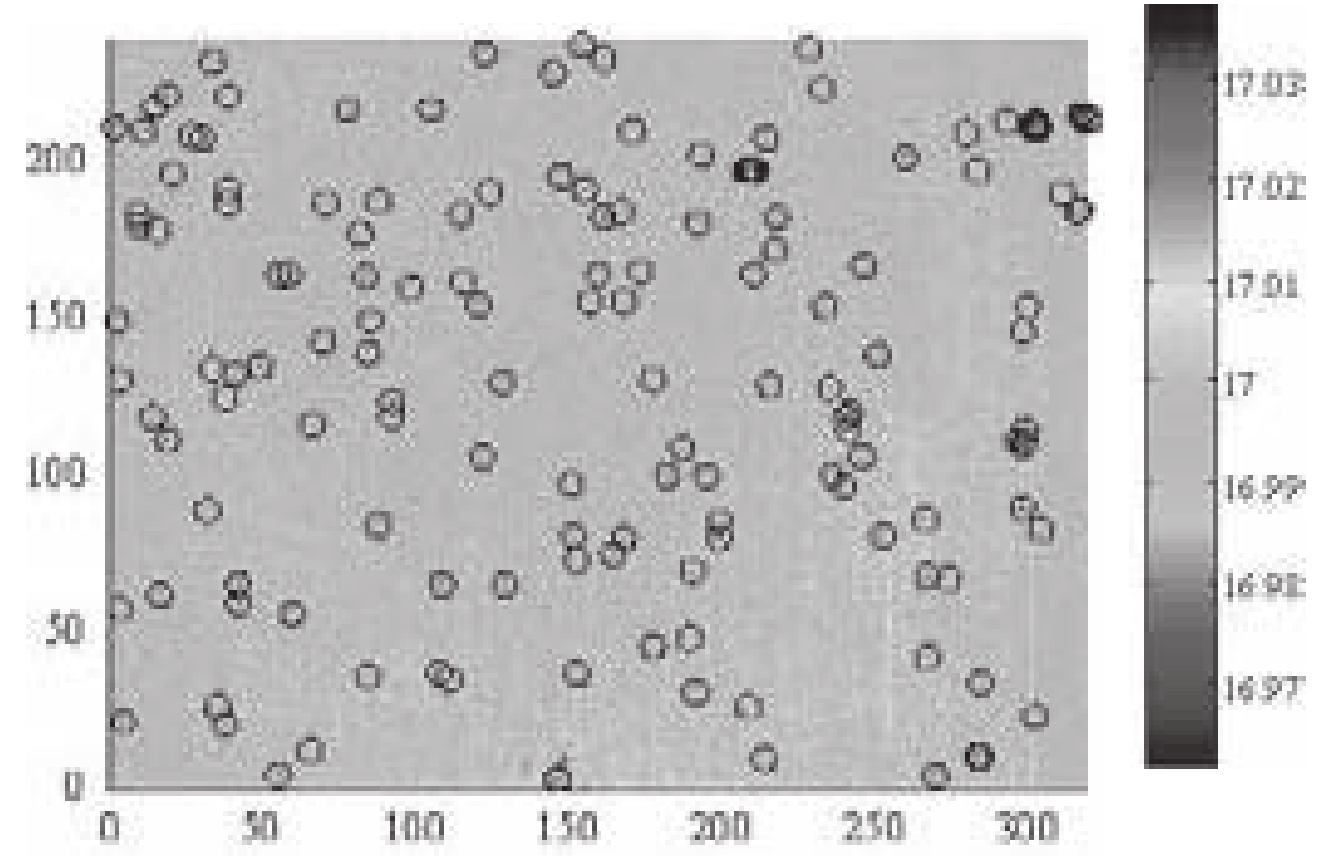

Figure 5. Example of bad pixel positions detected at a given $T_{j}^{B B}$

$$
T_{j}^{B B}=17^{\circ} \mathrm{C}, 154 \text { bad pixels. }
$$

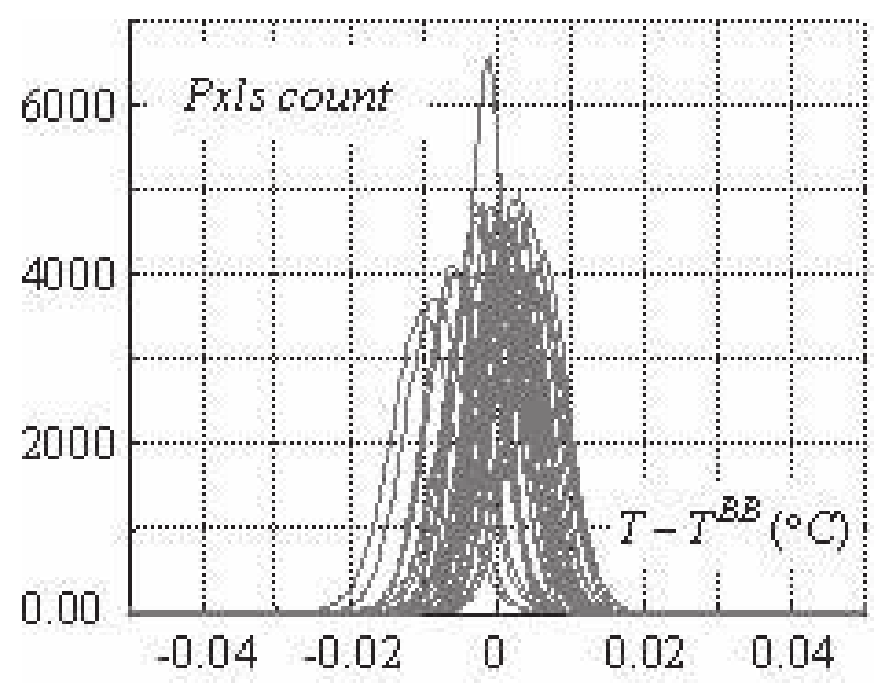

Figure 6. Histograms of pixel temperature differences for all $T_{j}^{B B}$.

In equation 10, the criterion eliminates pixels for which the maximal error is greater than the threshold $\delta T$. So the histogram construction of Figure 4 must be repeated for all $T_{j}^{B B}, j=1,2, . ., J$. Figure 6 shows the set of response distribution 
curves. The temperature range was defined by $T_{1}=17^{\circ} \mathrm{C}, T_{2}=34^{\circ} \mathrm{C}$ and we chose a regular thermal step $T_{j \sqcap 1}^{B B}-T_{j}^{B B}=0.5^{\circ} \mathrm{C}$.

When the scanning of the temperature range is over, the whole set of bad pixels can be established by joining sets determined for the different $T^{B B}$. The bad pixel percentage is $0.29 \%$ in the case shown here. Their positions are presented in Figure 7.

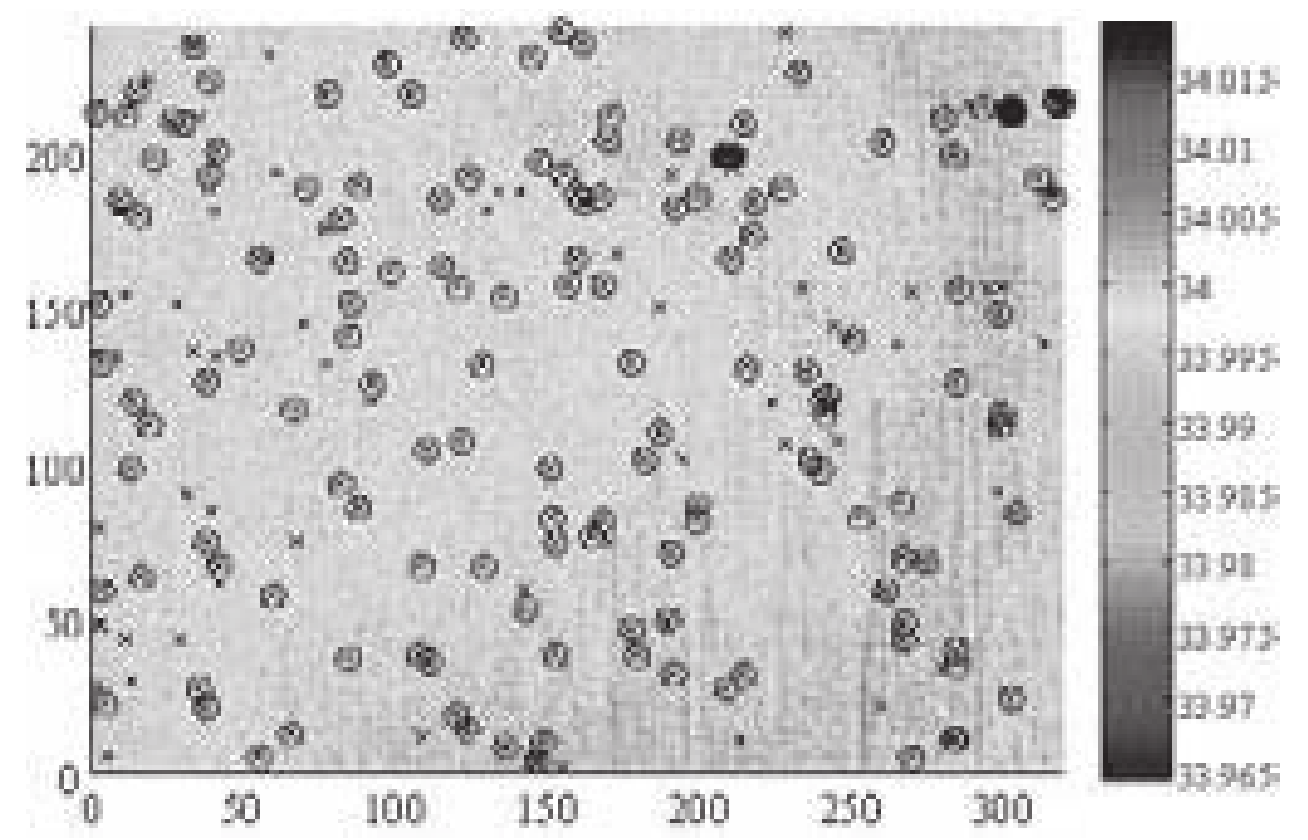

Figure 7. Bad pixel positions associated with [T1, T2]

It is also interesting to consider another representation of the pixel responses. In Figures 4 and 6, we have seen that the pixel responses approximately comply with a non-centred Gaussian distribution. The symmetry centre spread can be reduced if we change the criterion [10] for:

$$
\sup _{j=1,2, . ., J}\left|T_{i j}-\bar{T}_{j}\right| \geq \delta T
$$

where

$$
\bar{T}_{j}=\frac{1}{I} \sum_{i=1}^{I} T_{i j}
$$

This change is warranted by the fact that all the distribution function means stay within the interval $\left[-\delta T_{R},+\delta T_{R}\right]$ imposed by the limited performances of the black body. With this criterion, a new set of histograms was plotted in Figure 8.

Criterion [11] does not affect the number of bad pixels and thus it does not modify the calibration reliability. 


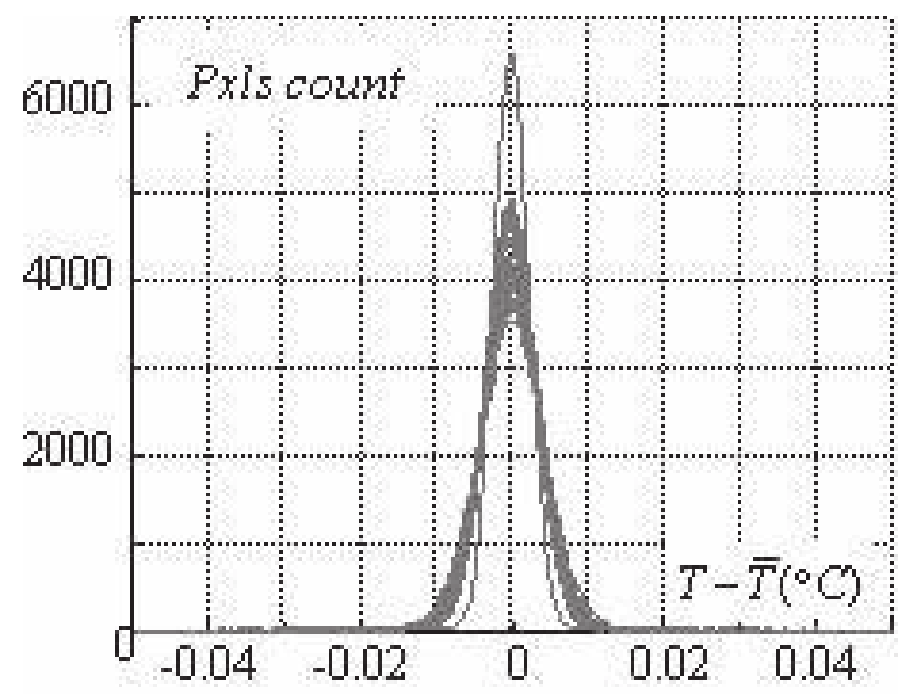

Figure 8. Temperature difference histograms computed for all $\bar{T}_{j}$.

\subsection{Other metrological aspects}

In the previous sections, we have provided an in-depth description of a calibration protocol limiting the errors made in estimating the thermal gradients and then heat losses occur by conduction. Naturally, other parameters may degrade the quality of the temperature measurements. We should underline here that the calibration and experiments must be performed after the IR camera has reached thermal equilibrium. This sometimes requires a waiting time of $4 \mathrm{~h}$. Naturally, this thermal equilibrium can be reached only if the testing room temperature is well regulated. Once the calibration is done for a given set of optical parameters, these must be blocked once and for all. For instance, if the optical focus is even slightly modified after calibration, a parasitic halo due to interactions between lens and detector increases the spatial homogeneity errors up to $0.1{ }^{\circ} \mathrm{C}$. Readers interested in these tricky metrological problems should be referred to (Pron et al., 2004). In what follows, we used the IRFPA camera to detect small temperature variations that accompany the deformation of polymers near room temperature. These small temperature variations are indeed a thermal signature of the thermomechanical behaviour of the deformed material. The next section describes the theoretical framework required for interpreting the thermal measurements and its associated calorimetric balance.

\section{PT and $\mathrm{Z}$ type models taking thermoelasticity into account}

As mentioned in section 1, we considered one-dimensional Poynting-Thomson and Zener type rheological models with thermoelastic branches replacing the elastic springs. For simplicity, the spectrum of each model was limited to only one relaxation time. All configurations were naturally considered in (Moreau 04). Here, we have eliminated those that give the same thermoelastic responses under the same 
loading conditions and those for which the dilatation parameters are not separately measurable using standard dilatometry techniques. The two remaining candidate models are plotted in Figure 9.

(a)

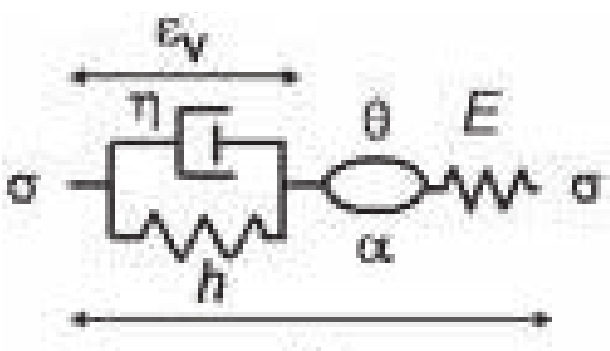

E (b)

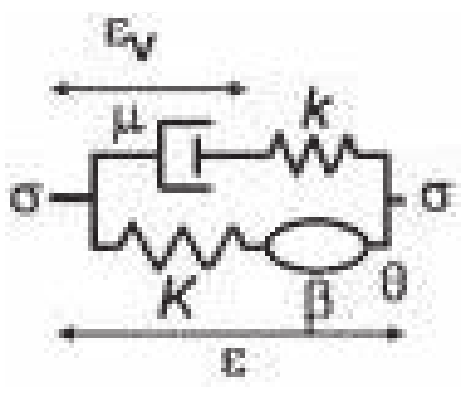

Figure 9. Basic sketch of $1 D$ viscothermoelastic models: (a) PT type; (b) Z type

For small perturbations, the state variables chosen for both models are: $\theta=T-T_{0}$ the temperature variation with respect to the room temperature (named $T_{0}$ ), $\varepsilon$ the tensile strain and $\varepsilon_{\mathrm{v}}$ the viscous part of $\varepsilon$. Our experimental observations indicated that the temperature variations remained low, so $\theta \ll T_{0}$. The $E, h, K, k$ constants are elasticity moduli, $\alpha$ and $\beta$ are linear thermal expansion coefficients, and $\eta$ and $\square$ are viscosity parameters. The material constants $\rho, C$ and $\kappa \square$ representing the density, the specific heat and the isotropic conduction coefficient, respectively, are still introduced. Both models belong to the classical framework of generalized standard models (Germain et al., 1983), so derivation of the heat equation then enabled us to specify the form taken by the different heat sources.

\subsection{PT type model}

With the above notations, the volume-free energy and the dissipation potential are as follows:

$$
\left\{\begin{array}{l}
\psi^{\mathrm{PT}}\left(\theta, \varepsilon, \varepsilon_{\mathrm{v}}\right) \square \frac{E}{2} \square-\alpha \theta-\varepsilon_{\mathrm{v}} \square^{2}-\left(\frac{E \alpha^{2}}{2} \square \frac{\rho_{0} C_{0}}{2 T_{0}}\right) \theta^{2}-s_{0} \theta \square \frac{h}{2} \varepsilon_{\mathrm{v}}^{2} \\
\varphi^{\mathrm{PT}}\left(q, \dot{\varepsilon}, \dot{\varepsilon}_{\mathrm{v}}\right) \square \frac{q \cdot q}{2 \kappa T_{0}} \square \frac{\eta}{2} \dot{\varepsilon}_{\mathrm{v}}^{2}
\end{array}\right.
$$

where $s_{0}$ is the specific entropy associated with the initial state $\left(\theta \square \varepsilon, \varepsilon_{\mathrm{v}}\right)=(0,0,0)$, and where $q$ is the heat influx vector. The heat diffusion equation can then be written as:

$$
\rho C \dot{\theta} \square \operatorname{div} q \square \eta \dot{\varepsilon}_{\mathrm{v}}^{2}-E \alpha T_{0} \dot{\varepsilon}-\dot{\varepsilon}_{\mathrm{v}}[,
$$


where the intrinsic dissipation $d_{1}^{\mathrm{PT}}=\eta \dot{\varepsilon}_{\mathrm{v}}^{2}$ and the thermoelastic source $s_{\text {the }}^{\mathrm{PT}} \square-E \alpha T_{0} \dot{\varepsilon}-\dot{\varepsilon}_{\mathrm{v}}[$. Equation 13 is classically derived by combining the local expression of the two principles of thermodynamics (Lemaitre et al., 1990) in the particular case of potentials defined by equation 12 . Since the dissipation potential does not depend on $\dot{\varepsilon}$, the stress is, by construction, written as:

$$
\sigma \square \psi_{, \varepsilon}^{\mathrm{PT}} \square E \square-\alpha \theta-\varepsilon_{\mathrm{V}}[.
$$

This equation allowed us to draw up experimental protocols to estimate the two parameters $\alpha$ and $E$. $\alpha$ was estimated by using dilatometry tests performed at very slow ramp heating so that the stress and viscous strain remained at zero:

$$
\left.\frac{\mathrm{d} \varepsilon}{\mathrm{d} \theta}\right]_{\sigma=0, \varepsilon_{\mathrm{v}}=0}=\alpha .
$$

We stress that to be able to interpret the structural data given by a dilatometer in terms of material characteristics, the temperature rate must be sufficiently low to ensure a suitable thermal equilibrium throughout the sample. This constraint eliminates the possibility of distinguishing instantaneous from delayed dilatation mechanisms.

In a second step, we estimated the elasticity modulus $E$ by considering the initial slope of a stress-strain curve corresponding to adiabatic loading performed at a sufficiently high strain rate. By neglecting the viscous strain, the intrinsic dissipation and the heat losses by conduction, equations 13 and 14 then give:

$$
\left.\frac{\mathrm{d} \sigma}{\mathrm{d} \varepsilon}\right]_{\varepsilon_{\mathrm{v}} \square 0, q \square 0} \square E\left(1 \square \frac{E \alpha^{2} T_{0}}{\rho C}\right) .
$$

\subsection{Z type model}

The volume free energy and the dissipation potential associated with the $\mathrm{Z}$ type model are as follows:

$$
\left\{\begin{array}{l}
\psi^{\mathrm{Z}}\left(\theta, \varepsilon, \varepsilon_{\mathrm{v}}\right) \square \frac{K}{2} \square-\beta \theta \square^{2}-\left(\frac{K \beta^{2}}{2} \square \frac{\rho_{0} C_{0}}{2 T_{0}}\right) \theta^{2}-s_{0} \theta \square \frac{k}{2} \square-\varepsilon_{\mathrm{v}} \square^{2}, \\
\varphi^{\mathrm{Z}}\left(q, \dot{\varepsilon}, \dot{\varepsilon}_{\mathrm{v}}\right) \square \frac{q \cdot q}{2 \kappa T_{0}} \square \frac{\square}{2} \dot{\varepsilon}_{\mathrm{v}}^{2}
\end{array},\right.
$$

where $s_{0}$ and $q$ have the same meaning as in the previous model. The corresponding heat equation is written as: 


$$
\rho C \dot{\theta} \square \operatorname{div} q \square \square \dot{\varepsilon}_{\mathrm{v}}^{2}-K \beta T_{0} \dot{\varepsilon}
$$

In the right-hand member of equation 18 , the intrinsic dissipation $d_{1}^{\mathrm{Z}}$ is $\square \dot{\varepsilon}_{\mathrm{v}}^{2}$ while the thermoelastic coupling source $s_{\text {the }}^{\mathrm{Z}}$ corresponds to $-K \beta T_{0} \dot{\varepsilon}$. With the irreversible part of the stress being zero, the stress is derived from the state equation:

$$
\sigma \square \psi_{, \varepsilon}^{\mathrm{Z}} \square K \llbracket-\beta \theta \square k \llbracket-\varepsilon_{\mathrm{v}}[.
$$

The dilatation coefficient $\beta$ was estimated using, as before, data extracted from dilatometry tests performed at very low ramp heating in order to ensure a mechanical and thermal equilibrium, so that $\sigma=0$ and $\varepsilon=\varepsilon_{\mathrm{v}}$, and hence:

$$
\left.\frac{\mathrm{d} \varepsilon}{\mathrm{d} \theta}\right]_{\sigma=0, \varepsilon_{\mathrm{v}}=\varepsilon}=\beta .
$$

The $K$ modulus was obtained using relaxation tests at different strain amplitudes. The $K$ modulus is the slope of the straight line which describes the correspondence between the stress level and the strain amplitude once the mechanical and thermal equilibrium is reached.

$$
\left.\frac{\sigma}{\varepsilon}\right]_{\varepsilon_{\mathrm{v}}=\varepsilon, \theta=0}=K .
$$

\section{Analysis of thermoelastic effects of PMMA and PC polymers}

These materials are very often used in industrial applications. Both polymers were provided by ATOFINA. We used standard dog bone shaped test specimens with the following gauge part sizes: length $(60 \mathrm{~mm})$, width $(10 \mathrm{~mm})$, and thickness $(4 \mathrm{~mm})$. The main thermophysical characteristics of both materials are grouped in Table 1. Note that the temperature $T_{\beta}$ corresponding to the first sub-vitreous relaxation peak of the PMMA is slightly greater than the room temperature at which the tests were performed $\left(\mathrm{T}_{0} \approx 20-24^{\circ} \mathrm{C}\right)$, while the relaxation peak temperature of the PC polymer is highly negative. Consequently, at around room temperature, the PC samples showed greater molecular mobility than the PMMA samples. Due to this property, the PC samples showed good ductility while the PMMA samples remained particularly brittle. As the glass transition temperature of both polymers is greater than $100^{\circ} \mathrm{C}$, they naturally remained in a glassy state during the deformation tests. Besides, the $\alpha$ and $\beta$ coefficients were measured under the same experimental conditions. Hence $\alpha=\beta$ even though a different thermodynamic path depending on the model is associated with a (very slow) dilatometry test (Equations 16 and 20). 


\begin{tabular}{|c|c|c|}
\hline & PMMA & $\mathrm{PC}$ \\
\hline $\begin{array}{l}\rho\left(\mathrm{Kg} \mathrm{m}^{-3}\right) \\
\text { (measured) }\end{array}$ & 1160 & 1190 \\
\hline $\begin{array}{c}C\left(\mathrm{~J}^{\mathrm{Kgg}}{ }^{-1} \cdot{ }^{\circ} \mathrm{C}^{-1}\right) \\
(\text { Atofina })\end{array}$ & 1450 & 1200 \\
\hline $\begin{array}{c}\kappa\left(\mathrm{W} \cdot \mathrm{m}^{-1} \cdot \mathrm{K}^{-1}\right) \\
\quad(\text { Atofina })\end{array}$ & 0.17 & 0.2 \\
\hline $\begin{array}{l}\alpha=\beta\left({ }^{\circ} \mathrm{C}^{-1}\right) \\
\text { (measured) }\end{array}$ & 7. $10^{-6}$ & 7. $10^{-6}$ \\
\hline $\begin{array}{c}E(\mathrm{MPa}) \\
\text { (measured) }\end{array}$ & 3650 & 2650 \\
\hline $\begin{array}{c}K(\mathrm{MPa}) \\
\text { (measured) }\end{array}$ & 3500 & 2570 \\
\hline $\begin{array}{c}T_{\beta}\left({ }^{\circ} \mathrm{C}\right) \\
\text { (Erhenstein 99) }\end{array}$ & 35 & -70 \\
\hline $\begin{array}{c}T_{\mathrm{g}}\left({ }^{\circ} \mathrm{C}\right) \\
\text { (Erhenstein 99) }\end{array}$ & $105-120$ & 145 \\
\hline
\end{tabular}

Table 1. Thermophysical properties of PMMA and PC

Figures 10 and 12 show a time-course presentation of the longitudinal temperature profiles $\theta(x, t)$ recorded by the infrared camera. Tensile stress was superimposed (black curve) in order to depict the $\theta$ profiles as a function of loading. When the regularity of the specimen geometry and the low material diffusivity were taken into account, we considered that the mean heat source over each cross-section was sufficiently representative of the material behaviour (Chrysochoos et al., 2000). The uniform character of the temperature profiles is indeed consistent with a homogeneous distribution of the sources, especially for materials with low thermal diffusivity.

The primacy of the thermoelastic effects over the dissipation can easily be checked: Figures 10 and 12 indicate a distinct cooling of the specimen during the loading stages and heating during the unloading stages, with the mean temperature over each load-unload cycle being approximately zero. The image processing methods used to estimate heat source patterns have already been thoroughly described in (Chrysochoos et al., 2000). A one-dimensional thermal diffusion model was used hereafter. Where $O z$ denotes the loading direction, the diffusion equation reads:

$$
\rho C\left(\frac{\partial \theta}{\partial t}+\frac{\theta}{\tau_{\text {th }}}\right)-k \frac{\partial^{2} \theta}{\partial z^{ \pm}}=w_{c h},
$$

where $\tau_{\text {th }}$ is a time constant characterizing heat losses perpendicular to the loading direction, $w_{\mathrm{ch}}^{\prime}$ is the overall heat source which here comes essentially from 
thermoelastic couplings. The time constant $\tau_{\text {th }}$ was estimated while considering the temperature variations in the middle of the sample gauge part during the return to thermal equilibrium. Hence, $w_{\mathrm{ch}}^{\prime} \approx s_{\text {the }}^{\Theta}$, where $s_{\text {the }}^{\Theta}$ is the thermoelastic source derived from infrared data.

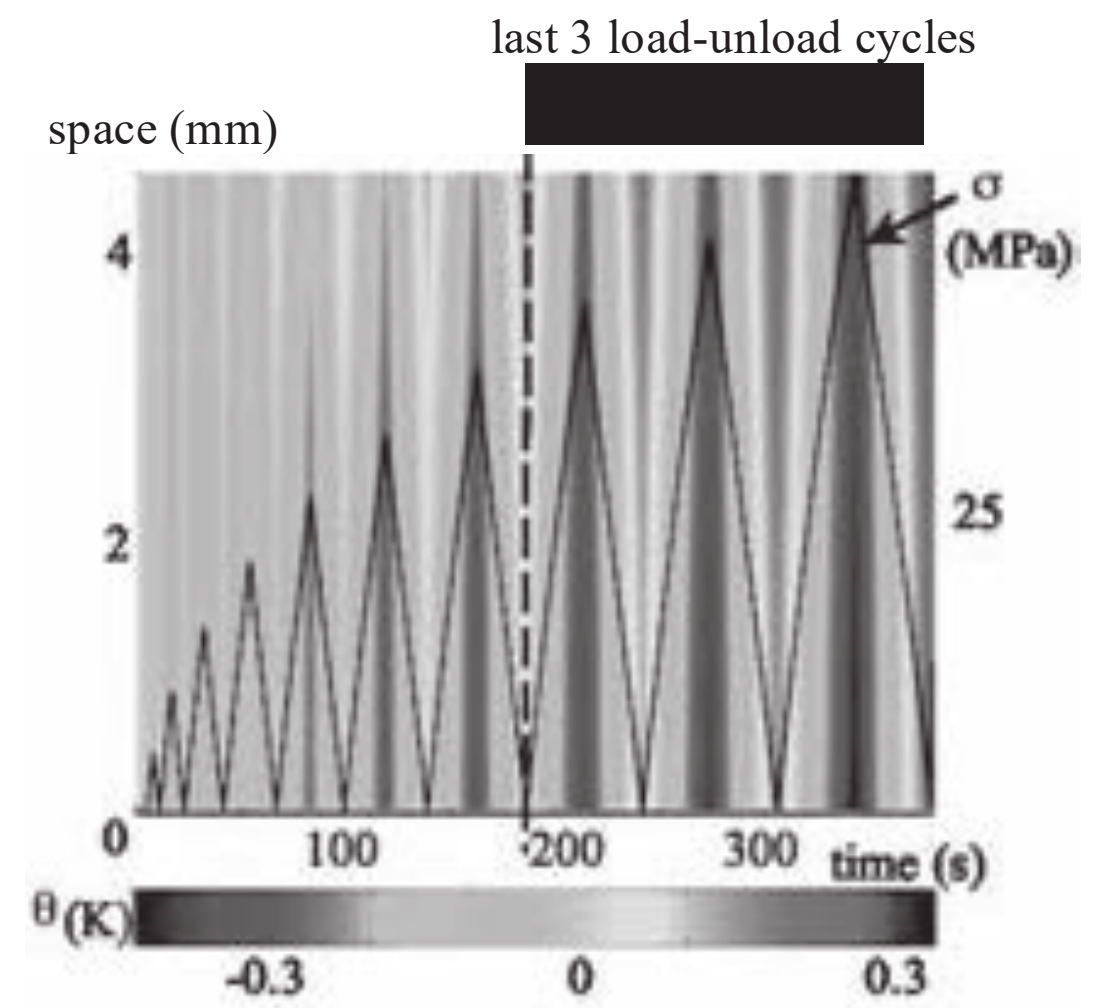

Figure 10. PMMA mechanical and thermal responses during a cyclic pulsating test.

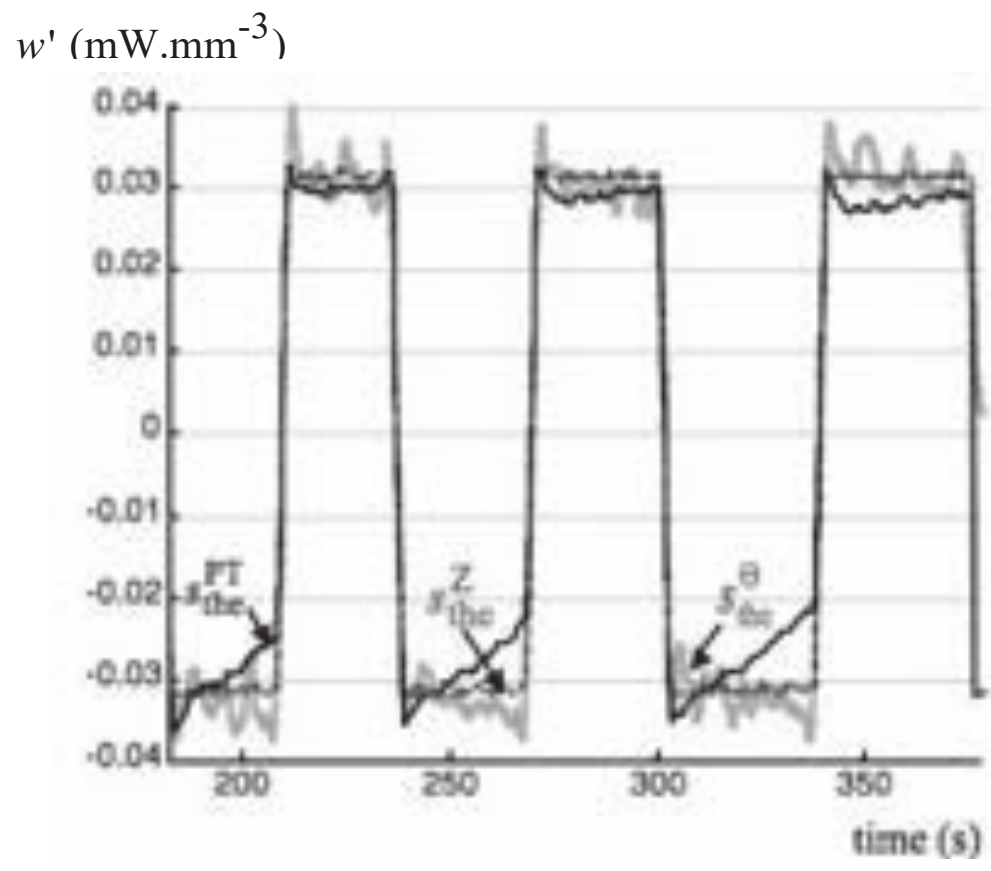

Figure 11. $s_{\text {the }}^{\Theta}, s_{\text {the }}^{\mathrm{Z}}, s_{\text {the }}^{\mathrm{PT}}$ patterns associated with the last 3 load-unload cycles (PMMA). 
In Figures 11 and 13, we plotted variations in $s_{\text {the }}^{\Theta}$, i.e. the thermoelastic source derived from the PT type model $s_{\text {the }}^{\mathrm{PT}}$ and from the Zener type model $s_{\text {the }}^{\mathrm{Z}}$. These two latter sources were computed using stress and strain measurements:

$$
s_{\text {the }}^{\mathrm{PT}} \approx-\alpha T_{0} \dot{\sigma}\left(E \alpha^{2} T_{0} / \rho C<4 \%\right) \text {, and } s_{\text {the }}^{\mathrm{Z}} \square-\beta T_{0} K \dot{\varepsilon} .
$$

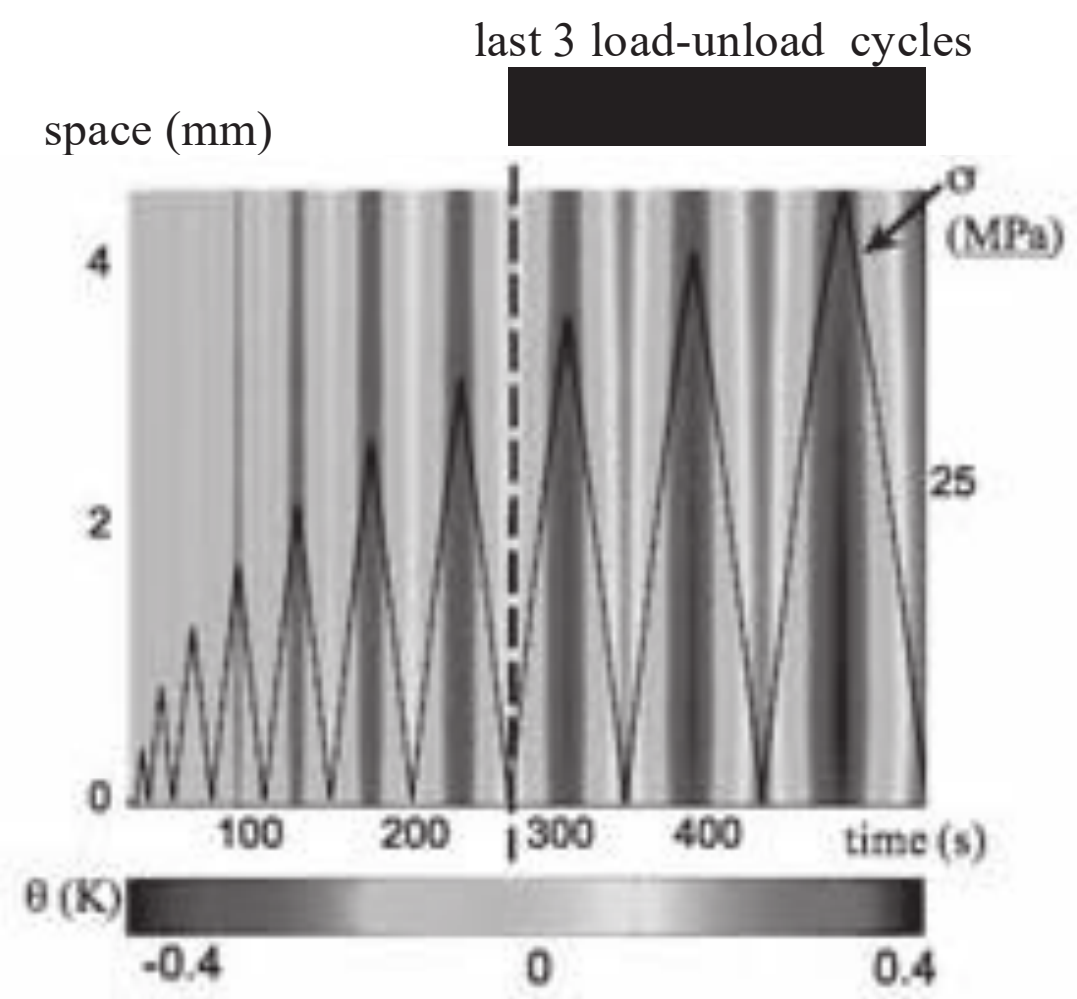

Figure 12. PC mechanical and thermal responses during cyclic pulsating tests.

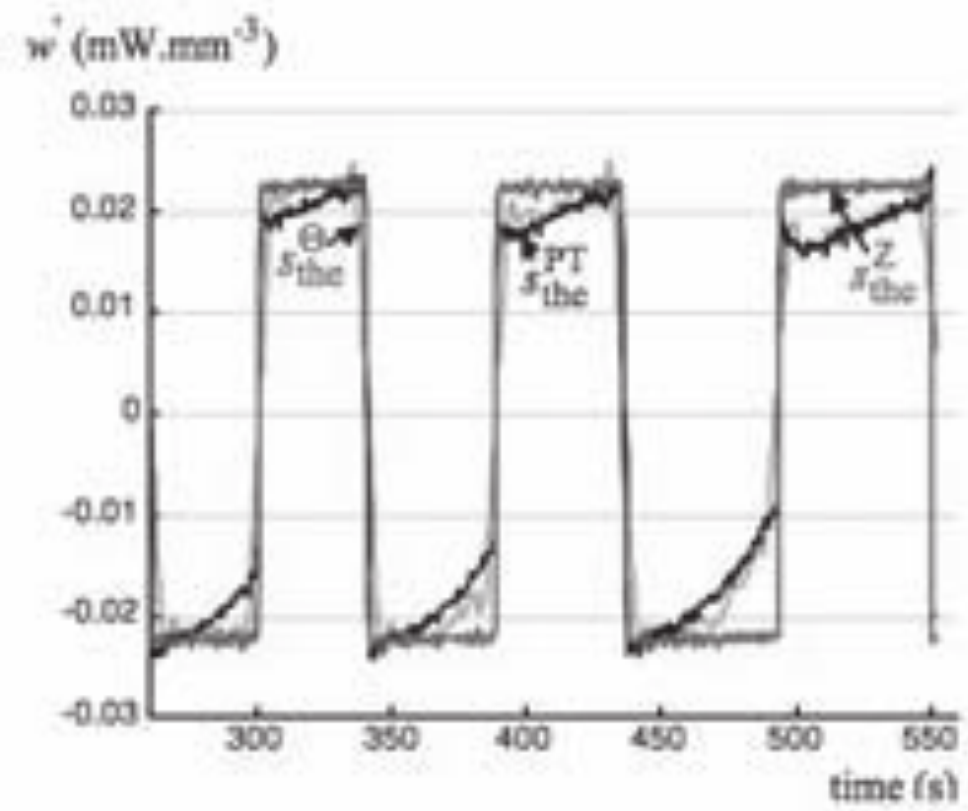

Figure 13. $s_{\text {the }}^{\Theta}, s_{\text {the }}^{\mathrm{Z}}, s_{\text {the }}^{\mathrm{PT}}$ patterns associated with the last 3 load-unload cycles, $(P C)$. 
Concerning the PMMA behaviour, Figure 11 shows that variations in $s_{\text {the }}^{\Theta}$ and $s_{\text {the }}^{\mathrm{Z}}$ remained close at all stress levels. Conversely, the source $s_{\text {the }}^{\mathrm{PT}}$ was quasi linear throughout the loading, while $s_{\text {the }}^{\Theta}$ remained almost constant. Regarding the PC behaviour, estimates of the three thermoelastic sources $s_{\text {the }}^{\Theta}, s_{\text {the }}^{Z}, s_{\text {the }}^{\mathrm{PT}}$ were plotted in Figure 13. A better prediction was obtained with the PT type model.

For the two materials studied, it should be noted that the order of magnitude of the thermoelastic source amplitude was about $60 \mu \mathrm{W} . \mathrm{mm}^{-3}$ whereas the dissipation intensity remained under $2 \mu \mathrm{W} . \mathrm{mm}^{-3}$.

\section{Concluding comments}

In this paper, we have proposed a calibration method of an IRFPA camera that avoids NUC and BPR operations and enables us to use the detector elements even in their nonlinear response domain. A polynomial calibration law was determined for each pixel. The accuracy of the temperature measurements is then limited by the planar black body performances used in the calibration.

We then applied this calibration method to analyse the thermomechanical behaviour of two vitreous polymers. Within a small perturbation framework, the thermographic experiment results led us to use viscothermoelastic models to describe the thermomechanical behaviour of PMMA and PC polymers. Indeed, from an energy standpoint, we observed that the thermoelastic effects predominated as compared to viscous dissipation.

In this setting, we noted a non-systematic equivalence between "series" and "parallel" models. This distinction appeared to be useful for describing differences in thermoelastic effects on PMMA and PC behaviours under the same loading conditions. A comparison of the thermoelastic sources, first derived from thermal data and secondly computed with mechanical measurements, showed that PMMA resembles a $\mathrm{Z}$ type model while PC resembles a PT type model. Complementary analyses are currently under way to determine if this behavioural difference is correlated with the intensity of molecular mobility.

\section{References}

Alfrey T.,Jr., Mechanical behaviour of high polymers, Vol. VI, Chap. B, pp. 93-233, Interscience Publishers, INC, New-York, 1948.

Atofina, Documentation, http://www.atofina.com/groupe/fr/f_elf_2.cfm.

Biot M. A., Mechanics of incremental deformations, Chap. VI, pp. 337-396, J. Wiley \& Sons Eds., 1965. 
Chrysochoos A., Louche H., "An infrared image processing to analyse the calorific effects accompanying strain localisation", International Journal of Engineering Sciences, 38, 2000, pp. 1759-1788.

Ehrenstein G.W., Montagne F., Matériaux polymères, Hermès Science Publications, 1999, pp. 302-319.

Ferry J. D., Viscoelastic properties of polymers, Chap. I, p. 1-32, J. Wiley \& Sons Eds, 1980.

Germain P., Nguyen Q. S., Suquet P., Continuum thermodynamics, Journal of Applied Mechanics, vol. 50, 1983, pp. 1010-1020.

Lemaitre J., Chaboche J.-L., Mechanics of Solid Materials, Cambridge University Press, 1990, Chap.II, pp.37-68.

Moreau S., Etude calorimétrique par thermographie infrarouge de la thermo-visco-élasticité de quatre polymères, $\mathrm{PhD}$ Thesis, Montpellier II University (France), 2004.

Papini F, Gallet P., Thermographie infrarouge, image et mesure, Masson Ed., 1994

Pron H., Bissieux C., "Focal plane array infrared cameras as research tools", Quantitative Infrared Thermography Journal, 1, 2, 2004, pp.229-240. 